\title{
The future of the International Schools for Young Astronomers (ISYA)
}

\author{
Itziar Aretxaga \\ ISYA Program Director \\ Instituto Nacional de Astrofísica, Óptica y Electrónica (INAOE), \\ Coord. Astrofísica, Luis Enrique Erro no. 1, \\ Sta. Mara Tonantzintla, Puebla, Mexico C.P. 72840 \\ email: itziar@inaoep.mx
}

\begin{abstract}
The 5-decade old ISYA program is evaluated in the context of the experience gathered in the field: 41 schools organized in 27 countries with a total of more than 1400 students to date. In the new era of fast internet connectivity, social media, virtual networks, big data and machine learning, the value of face-to-face graduate schools for regions with limited up-to-date astrophysics research is presented, together with the plan to develop the ISYA program into the next decade.
\end{abstract}

Keywords. IAU program: International School for Young Astronomers (ISYA), education, graduate school

\section{Introduction}

The ISYA program, established in 1967 by the IAU, organizes graduate-level threeweek-long intensive schools in countries where astronomy is not sufficiently developed, or in countries that can act as developing poles for those around them. The objective of ISYAs is to broaden the participants' perspective on astronomy by lectures from an international faculty on selected topics of astronomy, seminars, practical exercises and observations, and exchange of experiences, thus providing a network of ISYA fellow students and lecturers that will help students enhance their research opportunities in the near future.

The operation of the ISYA program has recently gone through restructuring. The Office for Young Astronomers (OYA) was created in 2015 as an alliance between the IAU and the Norwegian Academy of Science and Letters (NASL) to provide a sound financial and organizational basis for the ISYA program. The OYA is a virtual office operated by a Steering Committee, chaired by an IAU Vice-President. The Steering Committee oversees the ISYA program on behalf of the IAU and the NASL, and it reports regularly to them via their representatives. The ISYA Director and Deputy Director are jointly responsible for the operation of the ISYA program and are elected by the OYA Steering Committee. Their work involves soliciting ISYA host countries, developing and executing ISYA programs in collaboration with the local organizers and guarding the quality standards of the program.

At present, the OYA sponsors all transportation expenses for ISYA students and lecturers and the local organizers cover all local expenses.

In the 52 years of operation, the ISYA has held 41 schools in 27 countries (marked in blue in Figure 1) and reached more than 1400 students to date, with a sound record of achievements (see Gerbaldi 2019, for an analysis of participants and hosts). 


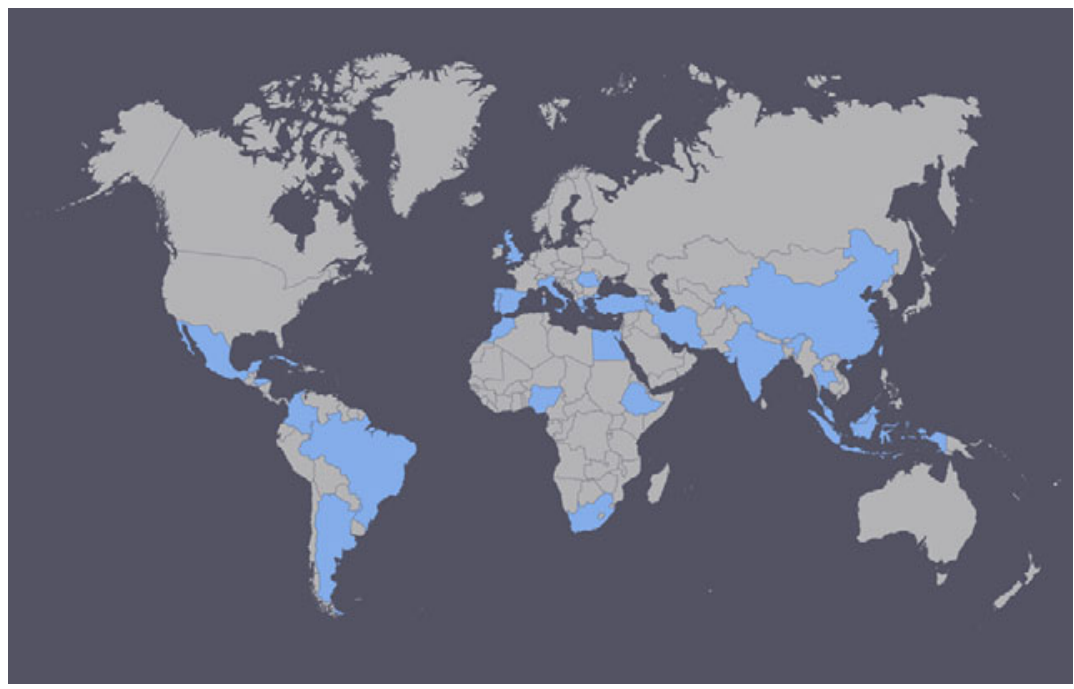

Figure 1. 52 years of history: 41 schools in 27 countries (marked in blue).

\section{The need for face-to-face tuition, some personal perspectives}

A first-world perspective on graduate school education includes access of interested students to an array of local world-experts on different topics through a graduate school program that, more often than not, offers scholarships during the years of education. Access to a good library, computer power, robust internet connectivity, downloads of data from international repositories, electronic access to papers in peer-reviewed journals, the possibility to attend international conferences and schools, teleconference facilities for collaboration meetings and remote observations are part of the granted research facilities for the young astronomer in a first-world country.

All or most of these conditions might be missing in the countries where ISYA serves its purpose of dissemination of research opportunities for the young astronomer in the developing world. As a prominent Kavli awardee who lectured in a recent ISYA put it "It is a humbling experience. I will tell my students they have nothing to complain about". We had no internet connectivity for most of the day in that particular school.

Talent, however, can be found anywhere in the world, and it is our duty and mandate to find ways to promote the tools that can help it flourish. This is particularly relevant from an historical perspective already in place when the ISYA program was established (Gerbaldi 2019), and key to meet the goals of the IAU Strategic Plan 2020-2030 in establishing professional astronomy forces in all countries around the world (van Dishoeck and Elmegreen 2018).

The ISYA schools give to the attending students unique opportunities that they often cannot find back in their home universities:

- Contact with an international panel of lecturers, who are active researchers in the topics they lecture on. Direct contact often results in looking closely at their topic of research together with the ISYA lectures, and on occasion long-term professional relations develop, including remote co-supervision of the students or recruitment of the students at the lecturers' universities.

- Topics that complement their basic astronomy background. Often students can have quality supervision in their home universities on a specific topic, but have no formal education on all other areas of astronomy. 
- Access to quality notes on selected basic topics on astrophysics. All lecture notes (often powerpoint presentations) are made available to the attending students. This material should be reinforced in future endeavours (see section 5).

- Knowledge of basic bibliographic and virtual observatory tools. More often than not attending students have not heard of tools such as the Astrophysics Data System, arXiv, or common data repositories.

- Some observational experience. Many students in developing countries do theoretical astrophysics work, and are unaware of the inherent biases and caveats in data acquisition and analysis.

- A network of fellow students to develop with into the new generation of astronomy professionals in the region.

- Knowledge of the opportunities they can access away from their countries of origin.

ISYA students usually regard their experience at the school as transformative. Although the backbone of the schools is an introduction to a broad selection of basic topics in astrophysics, with both formal "black-board" lectures and hands-on laboratories, the academic curriculum also includes a selection of workshops and discussion groups on career development inside and outside academia, CV writing, graduate school applications, paper writing, talk presentations, career stoppers such as impostor syndrome and unconcious biases, work ethics etc. Direct contact also allows for group activities such as development of short scientific projects during the school that enhance team-work skills and is a main bonding activity.

All of these items cannot be served by remote teaching. Often internet connectivity in the university and country of origin of the students is actually not stable enough for remote teleconferencing, anyway.

While the goal of ISYAs is to educate the students, education happens also for the lecturers. There is a continuous effort to find good-level lecturers (tenured or tenuretrack in their respective countries) who are familiar with the region, to encourage more interaction with the students post-school. Gender/ethnic balance is looked after, and we also look for a healthy rotation of new lecturers into the program that will become aware of the conditions in other parts of the world, and hence be sympathetic to the extra challenges that students and researchers in developing countries face.

\section{Relationship to other IAU sponsored schools}

Apart from the ISYAs, which are focused on graduate students, the IAU also organizes additional schools and training seminars at university level through the Regional Offices of Astronomy for Development (ROADs), others are supported as projects of the Office of Astronomy for Development (OAD) and by the Commission C1 (formerly 46) Astronomy Education and Development (Engvold 2018). Many of these schools target BSc students to encourage them to continue a scientific career. In recent years an increasing number of students coming from these bachelor-level schools to the ISYAs has been observed, and the outcome is a reinforcement and progression of the abilities of the students, a more determined view of completing a $\mathrm{PhD}$, and better awareness of the possibilities for advancing their careers inside and outside of academia.

\section{Plans for ISYAs in the near future}

Since 2017, the OYA has available funds for two schools every three years, and there are plans for expanding the program into two schools per year (van Dishoeck and Elmegreen 2018). The program has thus recently expanded, and a duty-cycle of 1 school every 2 years per developing IAU-region is planned for at present. The ISYA directors are committed to diversify the locations of the schools. Since hands-on observations can 
be replaced by remote observing and/or virtual observatory (VO) tutorials, there is no need to encourage applications from only host countries with available observatories. Good internet connectivity is needed for remote observations, and whenever this is not sufficient, pre-assembled archival data can be made available to the students. Figure 1 shows the grey landscape still to be conquered in the developing world for the ISYA program.

The program recognizes the need to promote astronomy in all its sub-disciplines, and introduce tools which regional students will likely do research with for their $\mathrm{MSc} / \mathrm{PhDs}$. Hence, the schools need to be tailored to the regional needs, and an intense dialogue between local organizers and ISYA directors has to ensue to warrant the success of the schools. Schools with emphasis on computational astrophysics, radioastronomy and/or high-energy astrophysics are being promoted together with the classical model based around an optical observatory. This will also open the interest of more countries to host an ISYA in the near future.

A case study in this sense is the Colombia school in July 2018, which had an emphasis on computational astrophysics, big data and machine learning, and high-energy astrophysics. The school was more heavily tilted towards these topics, each having at least one lecture course for its development, rather than a more extensive set of optical astronomy courses. An observational astronomy course was, nevertheless, also included in the program and one night of remote observations was successfully carried out (see Fig. 2). The benefit of such a school was obvious to our Colombian colleagues, as computational and high-energy astrophysics are current strongholds of their national astronomy development.

Apart from opening new ground in countries still to host ISYA schools, room has been made, and will still be made, for countries that need further reinforcement of their communities and for countries that can act as development poles for neighbouring countries, with a tradition to recruit students from neighbouring developing countries (e.g. parts of China, Mexico, South Africa,...)

\section{Strategies for the middle to long term}

There are avenues to find alliances with other educational initiatives both inside and outside the IAU-sponsored programs.

In Ethiopia 2017, for instance, the Square Kilometre Array South African Office offered a communication workshop to ISYA students on the last weekend of the school, and this was also supported by the regional OAD. The Colombia 2018 ISYA was co-organized by an alliance of Colombian universities and the regional OAD, and additional modules for high performance computing, machine learning and a science communication workshop were thus included in the program. In these workshops students developed OAD projectproposals (Ethiopia 2017, Fig. 3), or practised developing code and writing about their theses for the general public in Twitter, Facebook and Instagram (Colombia 2018). These complementary activities strengthen the notion that the schools have to be flexible in their curricula and adapted to local needs, opportunities and conditions.

Other partnerships could be sought with prominent observatories worldwide or with COSPAR to give further facilities to host countries in organizing ISYAs with concurrent funding.

\section{Proposed complementary initiatives}

The ISYA schools have an established dynamics that has proven beneficial in the last five decades and that has evolved through time to adapt to the current interconnected world. There are some initiatives that could make the program even more impactful. 

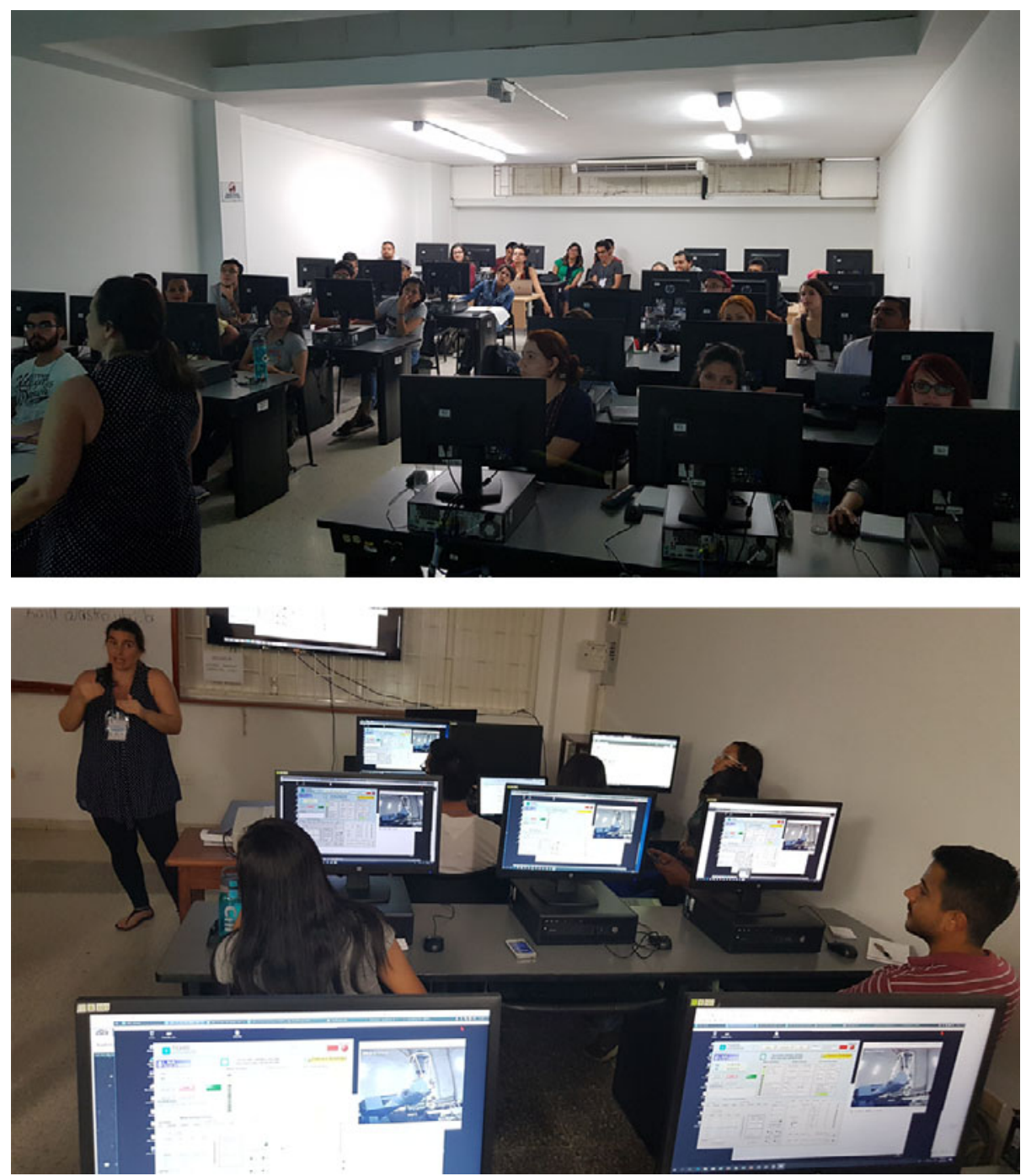

Figure 2. ISYA students at the premises of the Universidad Industrial Santander in Socorro, Colombia, using the 1.6-m Perkin-Elmer optical telescope of Observatorio Pico dos Dias in Minas Gerais (Brazil) in the evening of July 13th, 2018, coached by lecturer Karn Menndez Delmestre (Obs. Valongo, Brazil). Groups of students had the opportunity to enter, validate and command the observations during the session, and afterwards reduce and analyse them within their Observational Astronomy class.

a) Lecture Notes: Often research students in developing countries do not have access to good-quality textbooks. Their institutions simply cannot afford them.

Those that grew up in developing countries some decades ago remember with fondness the very cheap and excellent textbooks in physics and mathematics that Mir publishers in the ex-Soviet Union offered, and that students in developing countries could indeed purchase. Quality texts at a low cost are paramount to universal education. While the web is a big library, quality material is not easily found without breaking copyrights. Introductory 'copyleft' textbooks are an urgent need to promote further education in the developing world. It would be tremendously beneficial to the program to commission 


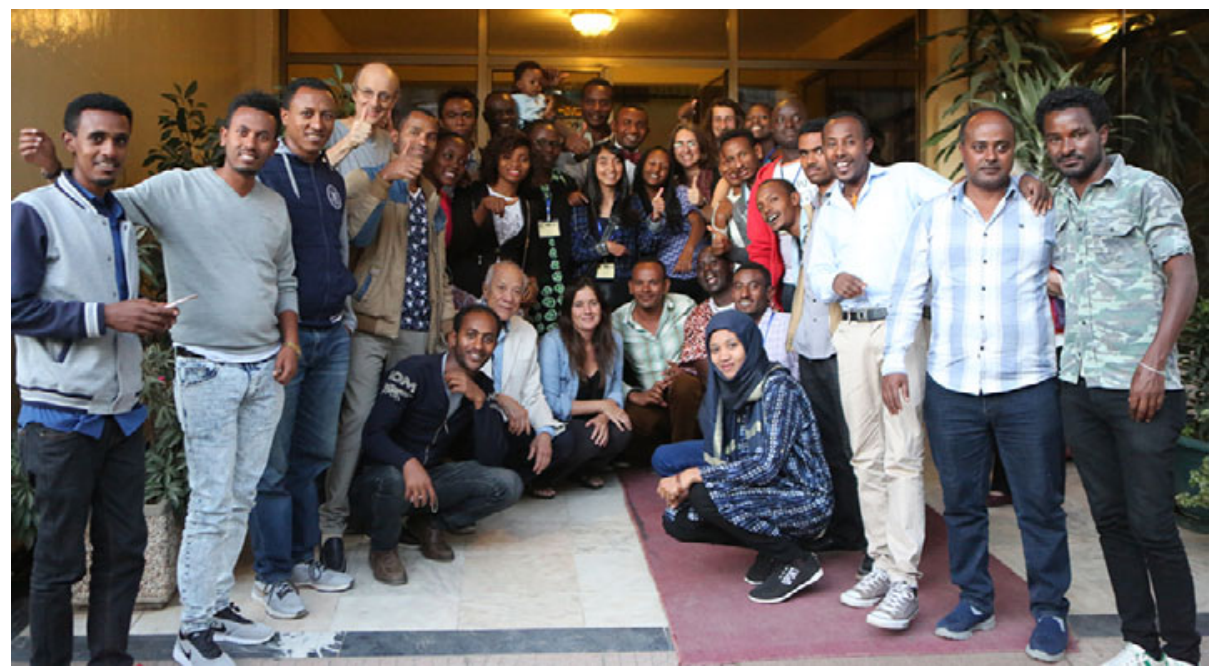

Figure 3. ISYA students and lecturers at the entrance of a hotel in Addis Ababa, Ethiopia, for the ISYA 2017. Notice the toddler at the back, son of one of the female students, being held by one of the lecturers. Two other female students declined joining the school due to insufficient means to care for their babies while they attended the school.

introductory astronomy textbooks (at the MSc level) that could be hosted in IAU web pages and offered to the world at large, free of charge. The textbooks could be either authored by prominent researchers that sign their chapters or the whole book, or be a collaborative text in the style of Wikipedia, but only by authorized specialists. The texts could be updated about every three years to maintain them as current and up-to-date with recent results. Texts on stellar evolution, radiative processes, planetary systems, the interstellar medium, galaxies, cosmology, optical astronomy, radioastronomy, X-ray astronomy... should be considered.

b) Graduate School Registry: Many universities have calls to recruit students around the world, and some national astronomy societies have a registry for studentships. Often the information does not reach promising students in the most remote areas of the world. It would be tremendously beneficial to ISYA students to have a platform for the registration of $\mathrm{MSc} / \mathrm{PhD}$ schools open to international students, which specifies in table format: Institution Name, Level of studies, whether there are grants available and which region of the world they welcome students from.

c) Family Mobility Grants: There is the need to seek funds for an IAU Family Mobility Grant. Many of the ISYA students and lecturers are young parents and many times they cannot leave their kids back home (for instance, while nursing), so they end up rejecting opportunities like the ISYA in order to tend to their families. The IAU should look into initiatives such as the AAS Dependent Care, to partially cover flight and accommodation expenses for a companion and kids during the school.

\section{Discussion}

DE AlbA-Martínez: In the Colombia case was the heritage component considered, since they have the oldest astronomical observatory in America?

AretXaGA: It was not. We emphasize heritage components through complementary talks when these are highlighted by the local hosts. In the Colombia school we highlighted the topics and tools that were identified by the local organizers as being the most important for developing astronomical research for the region. 
WADADEKAR: Although countries like China/South Africa/India are astronomically developed, a large section of the population does not have access to quality astronomy education. Surrounding countries also have many students who will benefit from ISYA. So, some ISYA schools should be organized in these countries.

ARETXAGA: I agree. We plan to maintain a double strategy of attracting new countries to host ISYAs and organizing them also in those countries that still could develop further, and in doing so, also help the surrounding countries.

\section{References}

Engvold, O. 2018, IAU's Interaction with Young Astronomers, this volume, 75-78

Gerbaldi, M. 2019, International School for Young Astronomers (ISYA) since the 1960s: Host Institutions and Participants, this volume, 445-451

van Dishoeck, E. \& Elmegreen, D. 2018, IAU Strategic Plan 2020-2030, www.iau.org/static/ education/strategicplan_2010-2020.pdf 